\title{
Dental arch changes from adolescence to adulthood in a spanish population: A cross-sectional study
}

\author{
Vera Paulino ${ }^{1}$, Vanessa Paredes ${ }^{2}$, Rosa Cibrian ${ }^{3}$, José-Luis Gandia ${ }^{4}$
}

${ }^{1}$ PhD in Dentistry. MS in Orthodontics. Private practice of orthodontics, Lisbon, Portugal. Collaborate Professor. Department of Orthodontics. Faculty of Medicine and Dentistry, University of Valencia, Spain

${ }^{2} \mathrm{PhD}$ in Dentistry. MS in Orthodontics. Doctor Professor. Department of Orthodontics. Faculty of Medicine and Dentistry, University of Valencia, Spain

${ }^{3}$ Professor. Department of Physiology. Faculty of Medicine and Dentistry, University of Valencia, Spain

${ }^{4}$ Professor. Director of Postgraduate Orthodontics Masters Course. Department of Orthodontics. Faculty of Medicine and Dentistry, University of Valencia, Spain

Correspondence:

Unidad docente de Ortodoncia.

Clínica Odontológica.

C/ Gascó Oliag $n^{\circ} 1$.

Valencia 46010. Spain.

verapaulino@hotmail.com

Received: $15 / 04 / 2010$

Accepted: $31 / 05 / 2010$

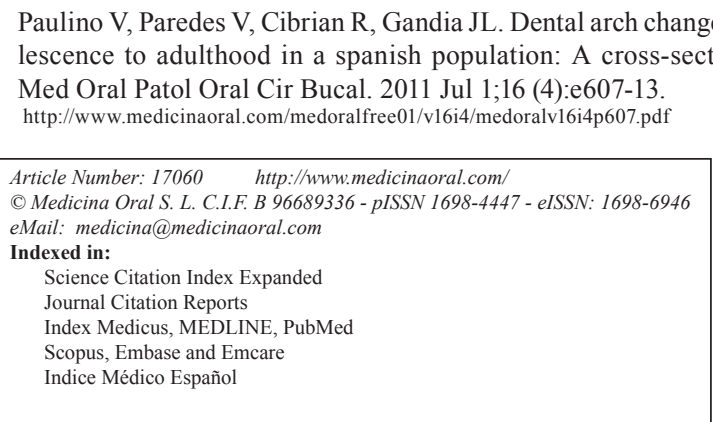

\begin{abstract}
Objectives: Given the controversy in the literature about the variations in intercanine and intermolar distances and arch perimeter once the eruption of permanent teeth is completed, the aims of this study were to assess the changes of these measures with age, analyzing its sexual dimorphism and variability in a Spanish population.

Study Design: 188 Spanish individuals distributed in three age groups were selected: 63 adolescents (mean age: 14.15 years), 62 young adults (mean age: 21.9 years) and 63 adults (mean age: 40 years). The intercanine and intermolar distances and arch perimeter were measured in each dental cast from each individual of the sample using a digital method. The results were compared between sex and age groups, the sexual dimorphism percentage (\%) of each measure and its variability coefficient (VC\%) were calculated.

Results: The results depend on sex and age and, therefore, these two factors will be analyzed jointly for each of them with the variation coefficient of the measurement. Intercanine and intermolar distances and arch perimeter were greater in men than in women, especially in the young adult and adult groups.

Conclusions: The intercanine distance and arch perimeter tended to decrease with age particularly in the female sex, whilst the intermolar distance didn't undergo significant changes. The intercanine distance is the dimension that presented the greatest variability, whereas the intermolar distance presented the least. The changes occur in the transition from adolescence (14 years of age) to adulthood (22 years of age) and the subsequent alterations are not relevant.
\end{abstract}

Key words: Arch dimensions, intercanine distance, intermolar distance, arch perimeter. 


\section{Introduction}

The dental arch undergoes various dimensional changes with age, the greatest alterations taking place during periods of growth. However, it is known that these changes do not cease with the onset of adulthood but continue at a slower rate, as it was demonstrated by studies carried throughout the life of an individual (1-4).

Several authors have observed an increase in intercanine and intermolar distances until permanent dentition is completed. The greatest increases take place during the period of greatest growth and decrease slightly from that moment on, especially in the lower intercanine distance $(5-13)$. In addition, others authors $(14,15)$ found that the intermolar distance remained stable. Other ethnic group's studies observed similar results (16-18).

Many authors $(1-3,5,7,15,19)$ have also obtained an increase in arch perimeter until permanent dentition is completed and a diminution of this dimension with age, mainly in the lower arch $(4,12)$.

As regards the different dimensions of dental arches between sexes, it can be observed that, generally speaking, males present greater arch dimensions than females $(2,6,9,10,20-23)$. Others studies found significant differences between sexes in different ethnics groups, in an Egyptian and Arab population (24) and in a Chinese population (25).

Due to the fact that most studies in Spanish populations focus on the first two decades of life and that there are no studies that evaluate later changes, the aims of this study were to assess the changes of these measures with age and to analyse its sexual dimorphism and variability. For that reason, we have selected patients of three age groups: adolescents, young adults and adults, with the aim of analysing the changes in the dental arch during the period of greatest growth (adolescents), during its final stage (young adults) and during the period of maturity in the fourth and fifth decades of life (adults). On the other hand, as we could observe, men and women present differences in dental arch. So, sexual dimorphism must be taken into account when individualizing orthodontic treatment as well as the variability between different individuals.

\section{Materials and Methods}

In the first place, it is worth noticing that this is a crosssectional study and not a longitudinal data.

The study sample was made up of individuals of both sexes, all of them Valencian, Spanish, resident in the city of Valencia, Spain, and with Valencian parents. The sample was homogenous and was divided into 3 chronological age groups: adolescents, young adults and adults.

The final sample consisted of 188 individuals:

1) 63 adolescents, with a mean age of 14.15 (age range: 1117 years of age). There were 34 females and 29 males.
2) 62 young adults, with a mean age of 21.9 (age range: 19-26 years of age), all students of the Faculty of Medicine and Odontology of Valencia. There were 23 females and 39 males.

3) 63 adults, with a mean age of 40 (age range: 31-50 years of age). In this group, 30 were female and 33 male. Group number 1) and 3) were recruited from the Orthodontics Clinic in the Faculty of Odontology of the University of Valencia.

The three types of the Angle molar class were present in all groups and none of the subjects had previously received Orthodontic treatment.

The material used in this study consisted of:

1. Plaster casts.

2. A conventional scanner for digitalising all study models.

3. Our own technology program, developed at our department, of which the reliability and reproducibility had been, tested previously (26). This program requires both a digitalised image of what one wishes to measure and back-up software for undertaking measurements.

The inclusion criteria for the study models were the following:

1. Presence of permanent dentition from first left-side molar to first right-side molar

2. Absence of alteration in the number of teeth.

3. Absence of abnormalities in dental size and shape.

4. Good quality of study models.

On each of the digitalised plaster casts, measurements were taken of intercanine and intermolar distances and arch perimeter in accordance with the following measurement criterion:

- Upper (UICD) and Lower Intercanine Distance (LICD) - the linear distance between the cusps of contra-lateral canines or, in the event of presenting evidence of wear, the distance between the centres of the worn surfaces.

- Upper (UIMD) and Lower Intermolar Distance (LIMD) - the linear distance between the points sticking out most from the molars measured on their vestibular faces.

- Upper (UAP) and Lower Arch Perimeter (LAP) - line that passes in the ideal mesial and distal contact points of each tooth from the first molar of one side to the first molar of the opposite side.

\section{-Statistical method}

The values found were introduced into a database for being processed, using the statistical package SPSS version 11.5 for Windows.

The Kolmogorov-Smirnov test was applied to determine which measurements showed normal distribution and to determine the type of statistics to be used.

For statistical analysis, the samples of each age group were grouped together per sex and the normality of the measurement of distribution was studied in each group using asymmetry analysis and Kurtosis. 
A comparison of two means was undertaken using Student's $t$ test and the differences of the means were estimated to a confidence interval of $95 \%$ (CI 95\%). Variance analysis was used to compare three or more means, and the Scheffe test was used for multiple comparisons. The percentage of sexual dimorphism was calculated. The variability of each dimension studied was evaluated by means of the variation coefficient (VC\%).

\section{Results}

Table 1 shows the Upper and Lower Intercanine Distance (UICD-LICD), the Upper and Lower Intermolar Distance (UIMD-LIMD) and the Upper and Lower Arch Perimeter (UAP-LAP) means for adolescents, young adults and adults of both sexes, the respective standard deviations (SD) and variation coefficients
(VC), while figures 1, 2 and 3 graphically represent these results.

The statistically significant difference in means between sexes in each age group was considered when the $\mathrm{p}$ value was greater than 0.05 , which is marked with an (*). Also statistically significant differences between age groups for each sex were considered $\mathrm{a}>\mathrm{b} \mathrm{p}<0.05$.

The results of intercanine, intermolar distances and arch perimeter depend on sex and age and, therefore, will be analysed jointly with the variation coefficient of the measurement. The percentage of sexual dimorphism in the arch distances (intercanine and intermolar) and arch perimeter were studied in table 2 . Since we did not find statistically significant differences in adolescents, the table just includes adults and young adults. This is the case where significant differences were found in the means between sexes.

Table 1. Upper and Lower Intercanine Distance (ICD), Intermolar distance (IMD) and Arch Perimeter (AP) for adolescents, young adults and adults of both sexes (female and male), the standard deviations (SD) and variation coefficients (VC). The statistically significant difference in means between sexes in each age group is marked with an $\left(^{*}\right)$. $a>b \quad p<0.05$ Difference between age groups for each sex.

\begin{tabular}{|c|c|c|c|c|c|c|c|c|c|c|}
\hline \multirow{2}{*}{ Sex } & & \multicolumn{3}{|c|}{ ADOLESCENTS } & \multicolumn{3}{|c|}{ YOUNG ADULTS } & \multicolumn{3}{|c|}{ ADULTS } \\
\hline & & Mean & SD & VC (\%) & Mean & SD & VC (\%) & Mean & SD & VC (\%) \\
\hline$F$ & UICD & $34.61 \mathrm{a}$ & 1.79 & 5.17 & $32.60 \mathrm{~b}$ & 2.09 & 6.41 & $33.35 \mathrm{~b}$ & 2.11 & 6.33 \\
\hline E & UIMD & $54.94 \mathrm{ab}$ & 2.15 & 3.91 & $53.61 \mathrm{~b}$ & 2.80 & 5.22 & 55.19 a & 3.20 & 5.80 \\
\hline M & UAP & $76.73^{\mathrm{a}}$ & 3.34 & 4.35 & $72.91^{\mathrm{b}}$ & 4.11 & 5.64 & $74.82^{b}$ & 4.02 & 5.37 \\
\hline A & LICD & 27.21 a & 1.68 & 6.17 & $25.34 \mathrm{~b}$ & 1.71 & 6.75 & $25.53 \mathrm{~b}$ & 1.64 & 6.42 \\
\hline $\mathbf{L}$ & LIMD & 52.78 & 1.73 & 3.28 & 52.08 & 2.91 & 5.59 & 52.41 & 2.76 & 5.27 \\
\hline L & LAP & $66.84^{a}$ & 3.53 & 5.28 & $62.71^{\mathrm{b}}$ & 3.91 & 6.24 & $63.88^{\mathrm{b}}$ & 3.64 & 5.70 \\
\hline & UICD & $27.21 \mathrm{a}$ & 1.68 & 6.17 & $25.34 \mathrm{~b}$ & 1.71 & 6.75 & $25.53 \mathrm{~b}$ & 1.64 & 6.42 \\
\hline $\mathbf{M}$ & UIMD & 55.29 & 2.46 & 4.45 & 56.56 & 3.39 & 5.99 & 56.99 & 2.75 & 4.83 \\
\hline A & UAP & 76.63 & 4.33 & 5.65 & 75.58 & 4.58 & 6.06 & 76.61 & 3.68 & 4.80 \\
\hline $\mathbf{L}$ & LICD & 26.58 & 1.35 & 5.08 & 26.38 & 2.21 & 8.38 & 25.95 & 1.85 & 7.13 \\
\hline E & LIMD & 53.22 & 2.27 & 4.27 & 54.07 & 3.55 & 6.57 & 53.74 & 2.56 & 4.76 \\
\hline & LAP & $67.46^{A}$ & 3.39 & 5.03 & $64.64^{\mathrm{B}}$ & 3.61 & 5.58 & $65.19^{B}$ & 3.32 & 5.09 \\
\hline
\end{tabular}




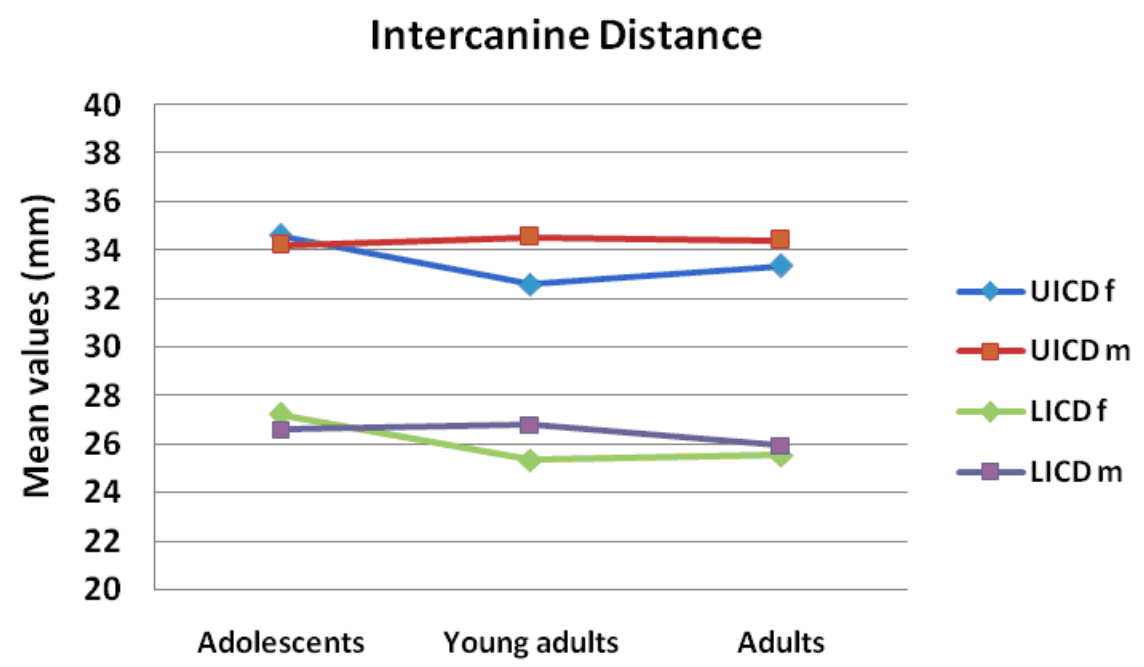

Fig. 1. Upper and Lower Intercanine Distance (UICD-LICD) mean values for adolescents, young adults and adults of both sexes (female and male).

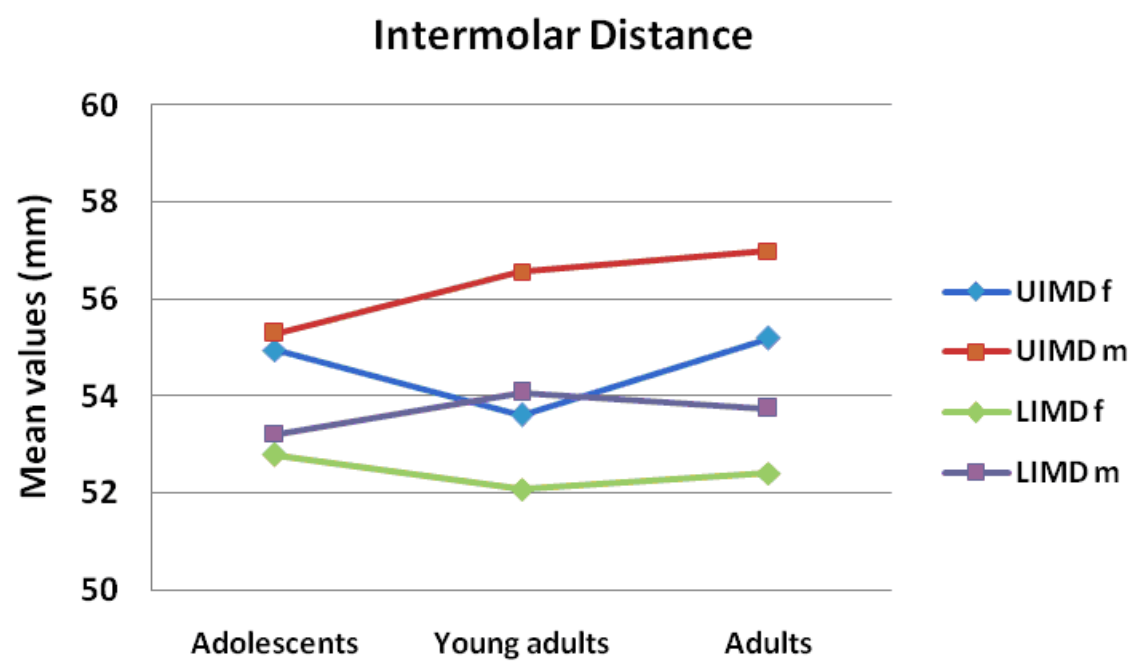

Fig. 2. Upper and Lower Intermolar Distance (UIMD-LIMD) mean values for adolescents, young adults and adults of both sexes (female and male). 


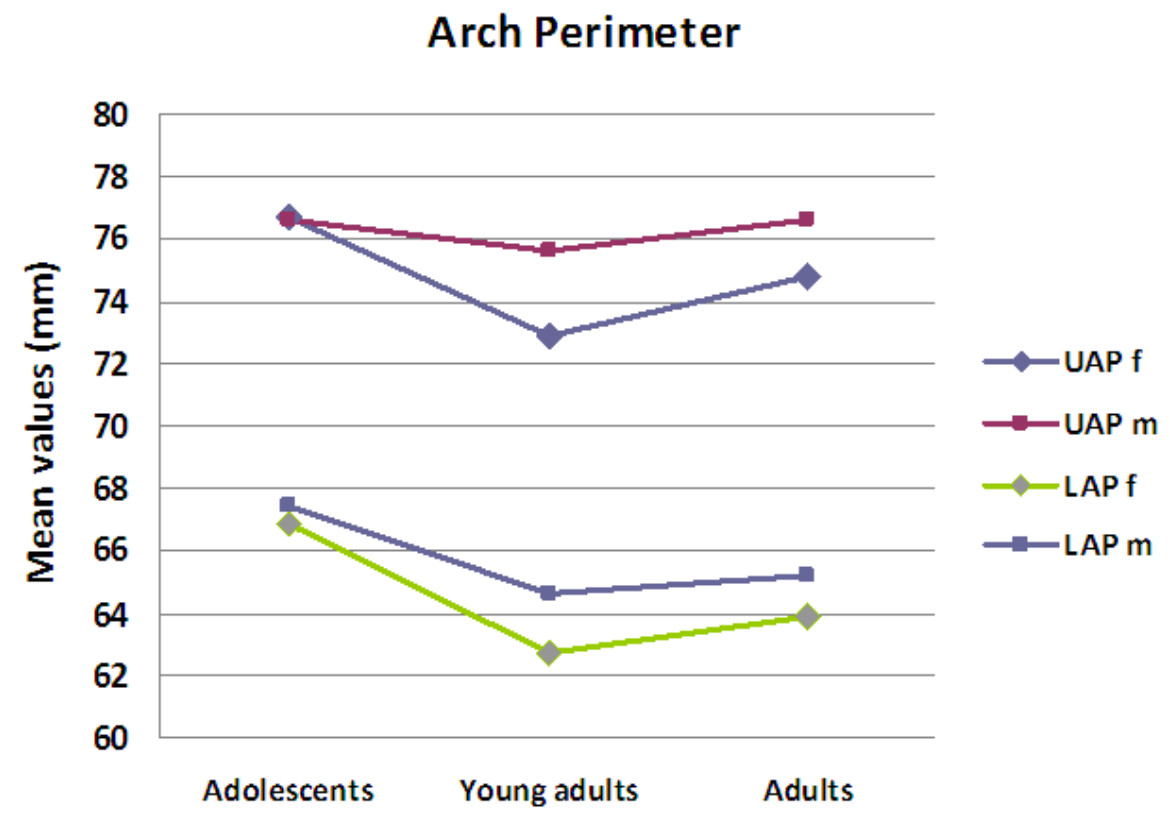

Fig. 3. Upper and Lower Arch Perimeter Distance (UAP-LAP) mean values for adolescents, young adults and adults of both sexes (female and male).

Table 2. Sexual dimorphism in the arch distances (Intercanine and Intermolar) and arch perimeter in young adults and adults.

\begin{tabular}{|c|c|c|}
\hline \multirow{2}{*}{} & \multicolumn{2}{|c|}{ Sexual dimorphism (\%) } \\
\cline { 2 - 3 } & Young Adults & Adults \\
\hline UICD & 5.9 & 3.1 \\
\hline LICD & 4.1 & 1.7 \\
\hline UIMD & 5.5 & 3.3 \\
\hline LIMD & 3.8 & 2.5 \\
\hline UAP & 3.7 & 2.4 \\
\hline LAP & 3.1 & 2.1 \\
\hline & & \\
\hline
\end{tabular}




\section{Discussion}

With regard to the ICD it can be said that, with the exception of adolescent males, the greater variability for this dimension ( $\mathrm{VC} \%$ ) was found in the lower arch, as did other study (22). Generally speaking, we found that there was greater variability of the ICD in males than in females.

As for the mean differences between sexes, in our study we did not find statistically significant differences in adolescents. The differences reached statistical significance in both arches for young adults and in the upper arch for adults. Our percentages of sexual dimorphism in young adults and adults coincide with some studies $(6,9,10,24)$ where greater intercanine distances were found in men.

In our work we have observed that the ICD tends to decrease significantly with age in females, between adolescents and young adults and between adolescents and adults. However, in males, even though a tendency towards a decrease between adolescents and adults can be observed, there are no significant differences between age groups. Our results are in line with the majority of studies $(5,15)$, where a decrease in ICD is observed with age after completing permanent dentition, especially in girls (15). Regarding the studies by others authors $(9-11,13,18,21)$ our results partly coincide with theirs, as they observed that the UICD diminished in both males and females. Both our work and the above mentioned studies contradict the Knott's (6) observations for whom the ICD remained stable after reaching permanent dentition, even in other ethnic group's studies (16-18).

Regarding the variability of IMD, with the exception of young adults, the greatest variability of VC (\%) was found in the upper arch, although the differences were very slight. Contrary to our results, Hashim and AlGhamdi (22) found a greater VC in the lower arch. We have found greater variability of IMD in males than in females in the adolescent and young adult groups. However, women presented greater $\mathrm{CV}$ in both arches in the adult group. In our study, we have observed higher values of variability in the intercanine distances than in intermolar distances.

As occurred with ICD, we did not find statistically significant differences between sexes in the adolescent group. Our results coincide with some authors $(2,9,10,24)$ who found greater values for intermolar distances in men. As for the difference of IMD between the different age groups, we have only found a significant increase in UIMD between young female adults and adults, without any significant differences between the other groups. On this subject, there is a great variety of results in the literature, some of which are contradictory. Thus, for some authors $(5,14)$ their sample presented a continuous decrease of the IMD. We partially coincide with Bishara et al. $(9,10)$, who found that alterations in the IMD were more modest than the alterations of the
ICD. Similar to our own results, these authors observed that the IMD tends to decrease between the ages of 13 and 26 and remain the same between 26 and 45 in females. Contrary to our results, in studies undertaken on patients in their third decade of life, researchers (11) observed significant decreases of IMD in both jaws and sex, while others (12) observed significant decreases of the LIMD and increases in the UIMD. We partly agree with some authors $(4,13)$, for whom the IMD did not undergo significant changes from the end of adolescence to the sixth decade of life.

There is a general tendency of AP diminution between adolescents and adults for the UAP in females and for the LAP in both sexes. However, the most significant decreases occurred for the lower arch between adolescents and young adults females.

Our findings partially coincide with others $(1-3,12,14)$, which contradicted Moorrees and Chadha (7) for whom AP were constant after age 14 .

With regard to the AP variability (VC), it can be observed that the greatest values in females were for the lower arch in all age groups, while in males this was the case for the upper arch, except in the adult group. Hashim and Al-Ghamdi (22) found the greatest variability in the mandible in the normoclusion group and, contrarily, in the maxilla in the malocclusion group. On the other hand, we have observed greater VC for males (except in adults) in the upper arch, while females presented the greatest variability in the lower arch.

Concerning to the differences between sexes, we did not find statistically significant differences in adolescents. In the other age groups, there were significant differences between males and females in both arches, the males presenting the biggest dimensions. Other authors $(2,21,23,24)$ made the same observation. We found higher percentages of sexual dimorphism in the young adult's upper arch.

Therefore, when drawing up an orthodontic treatment plan, it is important to know the characteristics of the dental arches, namely the arch widths (intercanine and intermolar) and perimeter, and their development over time, as this can provide us with information of the stability or relapse of our treatment.

\section{Conclusions}

1. We can conclude that the behaviour of intercanine and intermolar distance and arch perimeter is different with age, for each sex and dental arch. However, the changes, if any, occur in the transition from adolescence (14 years) to adulthood (22 years) and henceforth changes with age are not relevant.

2. The intermolar distance did not undergo significant changes with age after adolescence, with the exception of an increase in the upper arch between young adult and adult females. 
3. As for women, the intercanine distance and arch perimeter decrease in the transition from adolescence to adulthood, but in men this decrease is only seen in the perimeter of the mandible.

4. We therefore claim that the dental arch changes after the eruption of permanent dentition.

5. The intercanine and intermolar distances and arch perimeter were greater in men than in women, especially in the young adult and adult groups.

6 . The intercanine distance was the dimension that presented the greatest variability, whilst the intermolar dimension presented the least.

\section{References}

References with links to Crossref - DOI

1. Bishara SE, Treder JE, Damon P, Olsen M. Changes in the dental arches and dentition between 25 and 45 years of age. Angle Orthod. 1996;66:417-22.

2. Bishara SE, Jakobsen JR, Treder J, Nowak A. Arch length changes from 6 weeks to 45 years. Angle Orthod. 1998;68:69-74.

3. Eslambolchi S, Woodside DG, Rossouw PE. A descriptive study of mandibular incisor alignment in untreated subjects. Am J Orthod Dentofacial Orthop. 2008;133:343-53.

4. Dager MM, McNamara JA, Baccetti T, Franchi L. Aging in the craniofacial complex. Angle Orthod. 2008;78:440-4.

5. Slaj M, Jezina MA, Lauc T, Rajić-Mestrović S, Miksić M. Longitudinal dental arch changes in the mixed dentition. Angle Orthod. 2003;73:509-14.

6. Knott VB. Longitudinal study of dental arch widths at four stages of dentition. Angle Orthod. 1972;42:387-94.

7. Moorrees CF, Chadha JM. Available space for the incisors during dental Development-a growth study based on physiologic age. Angle Orthod. 1965;35:12-22.

8. Brown VP, Daugaard-Jensen I. Changes in the dentition from the early teens to the early twenties; a longitudinal cast study. Acta Odontol Scand. 1951;9:177-92.

9. Bishara SE, Jakobsen JR, Treder J, Nowak A. Arch width changes from 6 weeks to 45 years of age. Am J Orthod Dentofacial Orthop. 1997;111:401-9.

10. Bishara SE, Jakobsen JR, Treder J, Nowak A. Arch length changes from 6 weeks to 45 years. Angle Orthod. 1998;68:69-74.

11. Akgül AA, Toygar TU. Natural craniofacial changes in the third decade of life: a longitudinal study. Am J Orthod Dentofacial Orthop. 2002;122:512-22.

12. Tibana RH, Palagi LM, Miguel JA. Changes in dental arch measurements of young adults with normal occlusion--a longitudinal study. Angle Orthod. 2004;74:618-23.

13. Eslambolchi S, Woodside DG, Rossouw PE. A descriptive study of mandibular incisor alignment in untreated subjects. Am J Orthod Dentofacial Orthop. 2008;133:343-53.

14. DeKock WH. Dental arch depth and width studied longitudinally from 12 years of age to adulthood. Am J Orthod. 1972;62:56-66.

15. Sinclair PM, Little RM. Maturation of untreated normal occlusions. Am J Orthod. 1983;83:114-23.

16. Chen F, Terada K, Wu L, Saito I. Dental arch widths and mandibular-maxillary base width in Class III malocclusions with low, average and high MP-SN angles. Angle Orthod. 2007;77:36-41.

17. Arslan SG, Kama JD, Sahin S, Hamamci O. Longitudinal changes in dental arches from mixed to permanent dentition in a Turkish population. Am J Orthod Dentofacial Orthop. 2007;132:576.e15-21.

18. Henrikson J, Persson M, Thilander B. Long-term stability of dental arch form in normal occlusion from 13 to 31 years of age. Eur $\mathrm{J}$ Orthod. 2001;23:51-61.

19. Harris EF. A longitudinal study of arch size and form in untreated adults. Am J Orthod Dentofacial Orthop. 1997;111:419-27.
20. Knott VB. Size and form of the dental arches in children with good occlusion studied longitudinally from age 9 years to late adolescence. Am J Phys Anthropol. 1961;19:263-84.

21. Bishara SE, Treder JE, Damon P, Olsen M. Changes in the dental arches and dentition between 25 and 45 years of age. Angle Orthod. 1996;66:417-22.

22. Hashim HA, Al-Ghamdi S. Tooth width and arch dimensions in normal and malocclusion samples: an odontometric study. J Contemp Dent Pract. 2005;6:36-51.

23. Haralabakis NB, Sifakakis I, Papagrigorakis M, Papadakis G. The correlation of sexual dimorphism in tooth size and arch form. World J Orthod. 2006;7:254-60.

24. Abd-el Samad Younes S. Maxillary arch dimensions in Saudi and Egyptian population sample. Am J Orthod. 1984;85:83-8.

25. Ling JY, Wong RW. Dental arch widths of Southern Chinese. Angle Orthod. 2009;79:54-63.

26. Paredes V, Gandia JL, Cibrian R. New, fast, and accurate procedure to calibrate a 2-dimensional digital measurement method. Am J Orthod Dentofacial Orthop. 2005;127:518-9. 